\title{
MODELS AND METHODS OF PROCESSING OF INFORMATION ON LOADS OF ACOUSTIC SIGNALS IN TECHNICAL DIAGNOSTIC SYSTEMS
}

\author{
Artem Sharko \\ National Technical University of Kherson, Faculty of Cybernetics and Systems Engineering
}

Abstract. The paper presents one-dimensional discrete-continuous model of power spectrum estimation of the acoustic emission signal, that allows filtering the oscillating components of the acoustic emission signals. The mathematical formalism describing the environment was discussed, initiating the signals of acoustic emission. The problem of spectral analysis and synthesis of acoustic emission signals was solved with the help of Fourier transform. The dependence of acoustic vibrations spectra on the size of the medium parameters and microstructure has been discussed, as well.

Keywords: acoustic wave modeling, discretization

\section{MODELE I METODY PRZETWARZANIA INFORMACJI DLA SYGNALÓW AKUSTYCZNYCH W SYSTEMACH DIAGNOSTYKI TECHNICZNEJ}

Streszczenie. W artykule przedstawiony jednowymiarowy, dyskretno-ciagły model wyznaczania energii widma sygnatu akustycznego, który pozwala na filtrowanie jego składowych widmowych. Rozwiąano formalizm matematyczny opisujacy środowisko, inicjujacy emisję sygnałów akustycznych, w którym problem analizy widmowej i syntezy emisji sygnałów akustycznych zostal rozwiazany z pomoca transformaty Fouriera. Przedyskutowano także wplyw widma wibracji akustycznych na wielkość parametrów ośrodka i jego mikrostrukturę.

Słowa kluczowe: modelowanie fali akustycznej, dyskretyzacja

\section{Introduction}

The method of acoustic emission (AE) allows to conduct researches of kinetics by volume structural alteration on the different stages of materials deformation in real time. Physical nature of acoustic origin emission in materials is related to the processes of deformation and destruction [4, 5, 13, 17]. Models of the data processing in the system of determining defects by $\mathrm{AE}$ methods are based on the concepts of continuum environment and the continuum theory of dislocations, where the acoustic emission signal is indicative for a number of processes of the defect structure $[2,3,12]$. These processes are related to the movement of the dislocation the transition from elastic to plastic deformations, the extension of the dislocation loops and the annihilation of the separate sections of the structure $[11,15,16]$.

The relevance of studying the energy spectrum of acoustic emission (AE) signals of nanoscale objects is explained by the importance of solving questions concerning the characteristics of propagation of acoustic oscillations preceding the destruction of materials. To solve the problems of analysis and synthesis of materials with the given properties, a well-developed hierarchy of mathematical models is needed.

The generalized continua of the mechanics of a deformed solid are based on the concepts of the representative volume of the medium and the inclusion of rotational degrees of freedom. Models are constructed as deductive, so that their results act as consequences of axioms or postulates of a single system of assumptions. This provides the possibility of a consistent classification of theories according to the selected characteristics.

Mathematical models of data processing in system of definition of imperfections by AE methodare based on the discrete representations and the models of a continuous medium and the continual theory of the acoustic wave distribution where AE signal characterizes the variety of processes of defective structure of materials development.

Violation of the internal structure of materials initiates the occurrence of $\mathrm{AE}$ signals and their further propagation in an elastic medium. Dislocations are transformed into a far field, which propagates in the form of a wave packet independently at the speed of elastic waves in the absence of a stress source.

The main tasks of the paper are: development of the mathematical models of explaining mechanism of emission accumulation on the basis of the developing defects energy concepts; obtainment of the analytical expressions of interrelation of fracture parameters with the characteristics of $\mathrm{AE}$ signals through the functions of the applied tension.

\section{Formalization of the problem}

Theoretical explanation of the change in the structure of nanoscale objects develops in two ways: use of discrete models and continual ones. A characteristic feature of the energy spectrum of $\mathrm{AE}$ signal is the dual mechanism for the formation of $\mathrm{AE}$ signals: the discrete nature of structural changes and the continuous propagation of acoustic waves. All this makes it urgent to develop mathematical models of media with a microstructure.

In the model of a continuous medium in the form of a linear chain, the atoms interact by means of paired central forces connecting atoms and directed along a straight line connecting the centers of atoms. The instantaneous model of a continuous medium is based on the fact that the interaction between atoms remains paired, but it is realized through forces and moments. In the structural model of a continuous medium, its motion in space is described not by the coordinates and velocities of individual particles, but by the scalar density field and the vector field of velocities.

Behavior of this cell under load is characterized by interaction with the environment and is described by kinematic variables. Such models include, as information parameters of the structure, the dimensions and shape of the particles, on which the modulus of elasticity depends.

\section{Results and discussion}

One of the most important elements of this apparatus of mathematical physics is the concept of a quasi-continuum, which allows one to consider discrete and continuous models within the framework of a single formalism.

By a quasi-continuum we mean a one-dimensional $\mathrm{x}$-space and a class of admissible functions defined on it (Fig. 1).

It will assume that $a=$ const, independent of $n$. The value of the function at the nodes is denoted by $u(n)$. At points $x=n a$, the function $u(x)$ takes on the values $u(n a)$ which are the shifts of the oscillating point [9].

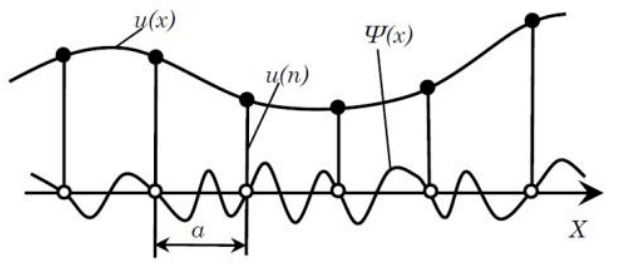

Fig. 1. One-dimensional quasicontinuum 
The elementary one-dimensional model of a discrete non-local microstructure can be presented in the form of an unlimited linear chain of the pointwise masses connected with elastic connections $\Psi$ (Fig. 2).

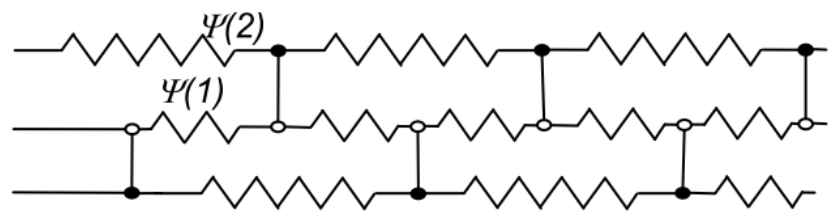

Fig. 2. Model of the discrete simple homogeneous structure: o-cooperating atoms of the basic chain; - - the nearest neighbours

The potential energy of such a chain is a functional from a field of displacement $u(n)$, causing elastic oscillations in the environment of [10]

$$
\begin{aligned}
\Phi=\Phi_{0} & +\sum_{n} \Phi(n) u(n)+\frac{1}{2} \sum_{n, n^{\prime}} \Phi\left(n, n^{\prime}\right) u(n) u\left(n^{\prime}\right)+ \\
& +\frac{1}{3 !} \sum_{n, n^{\prime}, n^{\prime \prime}} \Phi\left(n, n^{\prime}, n^{\prime \prime}\right) u(n) u\left(n^{\prime}\right) u\left(n^{\prime \prime}\right)+\ldots
\end{aligned}
$$

The difference of kinetic energy $T$ and a potential energy $\Phi$ defines Lagrange function $L$. If medium particles are effected by exterior forces $q(n, t)$ Lagrange function in harmonious approach expresses law of energy conservation and takes a form

$$
\begin{gathered}
L=\frac{m}{2} \sum_{n} \dot{u}^{2}(n, t)-\frac{1}{2} \sum_{n, n^{\prime}} \Phi\left(n, n^{\prime}\right) u(n, t) u\left(n^{\prime}, t\right)+ \\
+\sum_{n} q(n, t) u(n, t)
\end{gathered}
$$

Model parameters $\Phi(n), \Phi\left(n, n^{\prime}\right), \Phi\left(n, n^{\prime}, n^{\prime \prime}\right)$ are force constants. The force constants, defining the properties of such discrete model, are parameters of elastic connections between the particles.

The representation of function of one argument $\Phi(n)$ defines the elastic connections in a homogeneous linear chain.

$$
\Phi(n)=\Phi(-n)
$$

If $\Phi(n)$ is distinct from zero for $n>N$ each particle can cooperate with $N$-neighbours on the right and with $N$-neighbours on the left.

Taking into account Lagrange function the equation of an particles oscillated motion a linear chain will be the following

$$
m \ddot{u}(n, t)+\sum_{n^{\prime}} \Phi\left(n, n^{\prime}\right) u\left(n^{\prime}, t\right)=q(n, t)
$$

In the real mechanical systems long-range action is always limited by medium damping.

$$
\Phi(n)=-\Psi(n) \quad n \neq 0
$$

Taking this fact into account for the two particles $n$ and $n$, value of a potential energy $\Phi$ equals

$$
\Phi=\frac{1}{2} \sum_{n, n^{\prime}} \Phi\left(n, n^{\prime}\right) u(n) u\left(n^{\prime}\right)
$$

The kinetic energy of such pointwise masses chain for displacement $u(n, t)$, which depends on time equals

$$
T=\frac{m}{2} \sum_{n} \dot{u}^{2}(n, t)
$$

The elementary model of a complicated medium can be presented as a linear chain divided into elementary cells, each of which consists of two masses connected by elastic connections (Fig. 3).

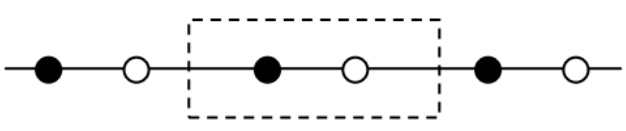

Fig. 3. Model of the discrete complicated structure
In this case the equation of the particles of $n$-th cell movement will be the following

$$
m_{j} \ddot{\omega}(n, j)+\sum_{n^{\prime} j^{\prime}} \Phi\left(n-n^{\prime}, j, j^{\prime}\right) \omega\left(n^{\prime}, j^{\prime}\right)=f(n, j)
$$

where $n$-cell number, $m_{j}(j=1,2)$ - a mass of particles in a cell, $f(n, j)$ - exterior force, $\omega(n, j)$ - displacement of $j$-th particle in $n$-th cell.

The wave of constant intensity can be the source of energy, which is lost at sudden decrease of rigidity. The wave concluded in an elastic forerunner leave from the front of destruction.

At metal straining the distance between the atoms under the influence of exterior forces varies, lines and the planes passing through atoms bent and at the expense of these facts the crystalline lattice is distorted. Elimination of the exterior forces between atoms again takes place in a crystalline lattice, and the material completely fills the volume it occupies.

Dislocations happen in places where the energy of activation, which is necessary for their formation, is reduced owing to the concentration of tension.

The energy demanded for the formation of the dislocation of one interatomic distance equals the energy, which is necessary for the formation of one vacant place in a lattice. In lack of other dislocations or imperfections it will move.

The transition from a discrete model to a continuum is done by extra-polarization of the functions given at discrete points by continuous fields of shifts and micro-turns. Depending on the number of expansion terms, we can consider different approximations of the discrete model of the structure of the medium and build a hierarchy of continual models.

The presence of internal connections between translational and rotational properties of a continuous medium is manifested in their connection with oscillatory properties.

In the mechanics of a continuous medium, the figure, which limits the region of structural changes during the initial stage of crack formation, has rotational symmetry if it passes into itself with all rotations. An analogue of such models is Cosserat model (Fig. 4).

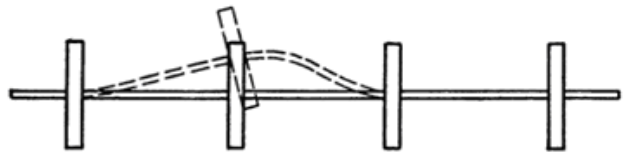

Fig. 4. Cosserat model

For this, in the model of a continuous medium that initiates the occurrence of AE signals (Fig. 5), it is necessary to introduce variables: relative displacement of particles inside the cell (9) and displacement of the cell center of mass (10) .

$$
\begin{aligned}
& \eta(n)=\frac{m_{1} \xi_{1} w(n, 1)+m_{2} \xi_{2} w(n, 2)}{I} \\
& u(n)=\frac{1}{m}\left[m_{1} w(n, 1)+m_{2} w(n, 2)\right]
\end{aligned}
$$

where $m_{1}$ and $m_{2}$ are the masses of the atoms in the cell, $I$ is the moment of the cell inertia, $\xi_{1}$ and $\xi_{2}$ are the coordinates of the particles in the cell with correspondence to the coordinate of the mass center, $m=m_{1}+m_{2}, I=m_{1} \xi_{1}^{2}+m_{2} \xi_{2}^{2}$.

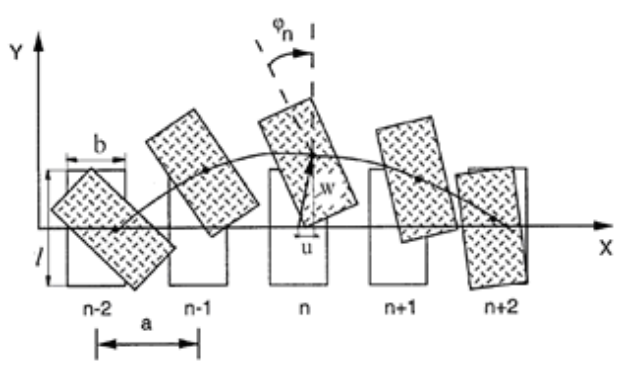

Fig. 5. Model for initiating acoustic emission signals 
Equation of motion

$$
\begin{aligned}
& m \ddot{u}(n)+\sum_{n^{\prime}} \Phi^{00}\left(n-n^{\prime}\right) \eta\left(n^{\prime}\right)+\sum_{n^{\prime}} \Phi^{01}\left(n-n^{\prime}\right) \eta\left(n^{\prime}\right)=q(n) \\
& I \ddot{\eta}(n)+\sum_{n^{\prime}} \Phi^{10}\left(n-n^{\prime}\right) \eta\left(n^{\prime}\right)+\sum_{n^{\prime}} \Phi^{11}\left(n-n^{\prime}\right) \eta\left(n^{\prime}\right)=\mu(n)
\end{aligned}
$$

Here, the matrix $\Phi^{s s t}(n)\left(s, s^{\prime}=0,1\right)$ is coordinatewise expressed in terms of the power constants $\Phi\left(n, j, j^{\prime}\right)$ of the precursors of AE signal [8].

The use of information and structural modeling to analyze the spectrum of $\mathrm{AE}$ signals of precursors of occurrence of internal stresses in a material is based on Fourier transforms of AE signals.

Fourier transform preserves the signal energy. It is meaningful only for signals of finite duration, the energy of which is finite. The spectrum of such initial signals rapidly approaches zero. These provisions are in full accordance with the physical meaning of the acoustic emission phenomenon.

The graph of the complex numbers argument values is the phase spectrum, and the modulus graph is the amplitude spectrum. One of the main properties of Fourier transform is the independence of the amplitude spectrum from the time shift of the signal, since when the function moves only its phase spectrum changes.

Fourier image of a real signal has symmetry: the amplitude spectrum is always an even function. This allows us to reduce complex functions and their Fourier-images to simpler ones. The spectrum of the total time function is equal to the sum of the spectra of its components. In Fourier transform AE signal is distributed to a basis of sines and cosines of different frequencies.

When constructing the model of the energy acoustic emission signals spectrum, the following assumptions are introduced:

- locality, i.e. limiting of structural changes in the environment;

- dynamism of processes;

- the signal to the radiation point is a pulsed Poisson process;

- Fourier transform of AE signal has stationary characteristics.

The displacement of elementary masses caused by structural changes in a one-dimensional chain of particles connected by elastic bonds $\Psi(x)$ initiates a propagating wave:

$$
u(x, t)=A e^{i(k x-\omega t)}
$$

The coefficients of Fourier transform are found by computing the scalar product of the signal with complex exponentials:

$$
F(\omega)=\int_{-\infty}^{+\infty} f(t) e^{-i \omega t} d t
$$

where $f(t)$ is the signal, $F(\omega)$ is Fourier transform.

The shape of the propagating AE signal depends not only on the displacement time $t$, but also on the frequency $\omega$. Therefore, in addition to the displacement functions in the time $u(t)$, we should consider their Fourier-images $u(\omega)$ related by the relations:

Their Fourier-images $u(\omega)$ related by the relation:

$$
\begin{gathered}
u(\omega)=\int u(t) e^{i \omega t} d t \\
u(t)=\frac{1}{2 \pi} \int u(\omega) e^{-i \omega t} d \omega
\end{gathered}
$$

Fourier transform has a number of drawbacks due to the fact that the individual features of the signals cause minor changes in the frequency image and are smoothed over the entire frequency axis.

Part of the problem of spectral analysis and synthesis of $\mathrm{AE}$ signals can be solved by means of a window Fourier transform

$$
F(\Omega, b)=\int_{-\infty}^{+\infty} f(t) \omega(t-b) e^{-j \Omega t} d t
$$

In this case, the operation of multiplying a signal by a window $(t-b)$, which is a local function moving along the time axis, is used. Then the transformation becomes time-dependent and the frequency-time description of the signal is realized.

\section{Fourier-transform experimental data}

The results of experimental studies on the establishment of the interconnection between the appearance of AE signals and the parameters of the force field for various loading stages of St3 (Fig. 6, 7).

Identification of structural features of material damage accumulation from $\mathrm{AE}$ data was carried out on the basis of an analysis of the degree of deformation of samples obtained from tests on a breakaway machine UM5.

Transformer oil was used as the contact material between the $\mathrm{AE}$ converters and the sample. The measuring unit used broadband sensors to the AF-15 acoustic and emission instrument. The information and measuring system used in the experiment provided the indication, registration and pre-processing of $\mathrm{AE}$ signals with their further storage in the memory of computer for subsequent post-processing of the received data and their real-time visualization using the RIGOL DS1052E Digital oscilloscope.
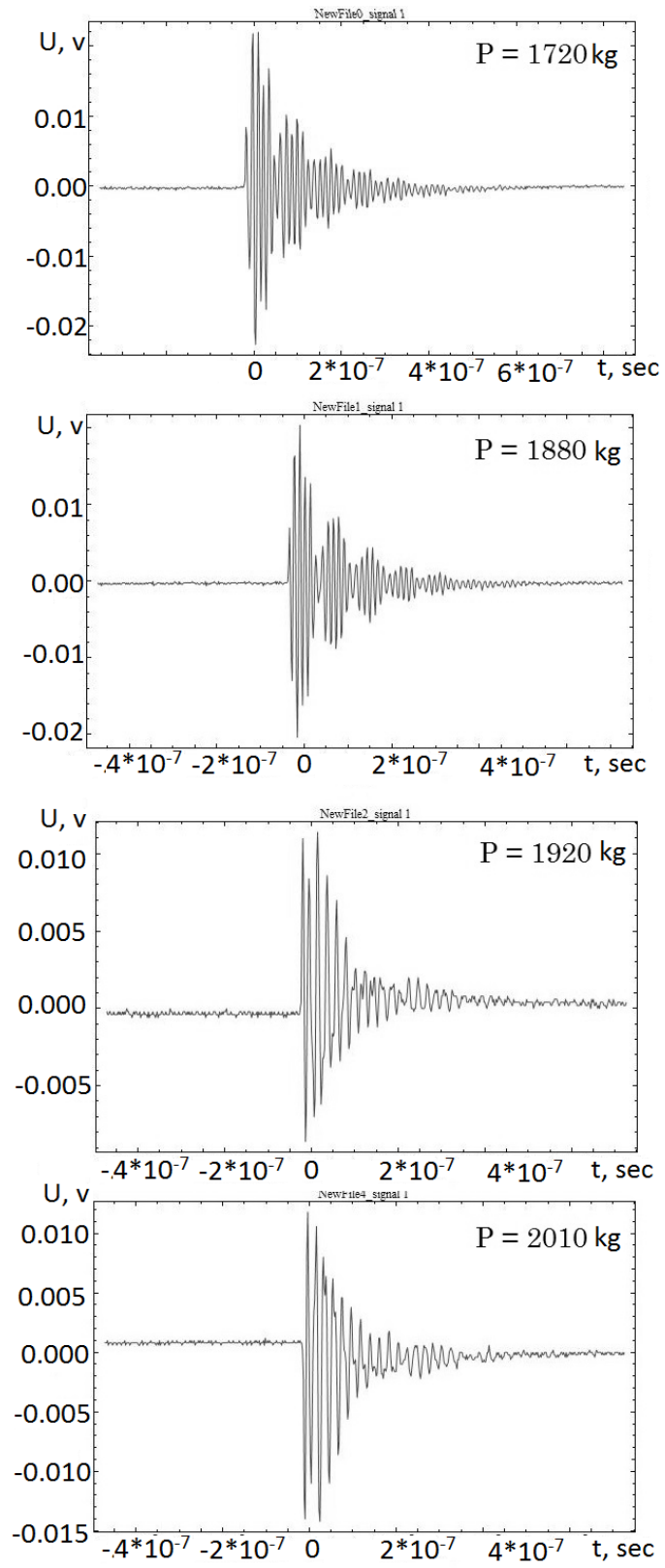

Fig. 6. Amplitude time distribution of spectral density of AE signals, under variou. loads 

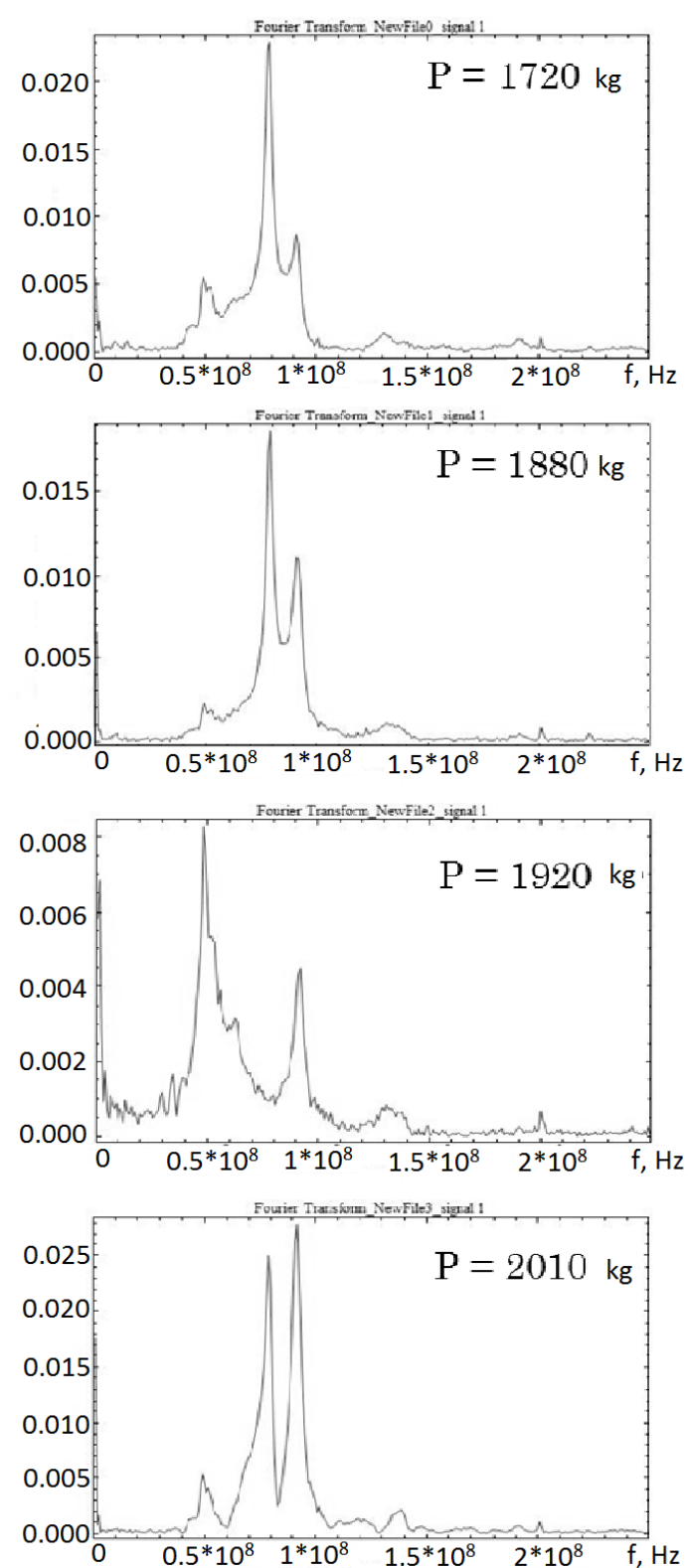

Fig. 7. Fourier-transform experimental data

When processing the experimental data, Mathematica 9.0 computer mathematics system and algorithms for working with numerical data arrays were used: finding the maximum (minimum) elements of the array, sorting the data of the array by the characteristic, combining the data, spline interpolation.

The density distribution of $\mathrm{AE}$ signals was determined by calculating the ratio of the number of intersections of the zero level of AE signal form to the time of its existence. The effect of a significant decrease in the distribution density of AE signals with increasing load is detected and quantitatively confirmed.

\section{Conclusions}

Compression of AE signal in time leads to an expansion of its spectrum, while shifting of AE signal in time, causes a phase shift of the spectrum proportional to the frequency. At the same time, the fundamental condition of translational and rotational invariance is observed in accordance with transformations of $\mathrm{AE}$ signal.

The presented results of the theoretical and experimental study of the energy spectrum of acoustic emission (AE) signals in the models of a continuous medium, the informational parameters of which are the operators of elastic energy, showed that the violation of internal bonds between translational and rotational properties of the continuous medium model in the form of a diatomic cell connected by elastic bonds initiates the oscillatory properties of the precursors of the destruction of materials of structures that are under load.

In the information and structural model of precursors of the appearance of acoustic emission signals, the oscillations of particles in a cell are characterized by the high-frequency component of $\mathrm{AE}$ signal, while the center-of-mass oscillation is characterized by its low-frequency component. Increasing the size and structure of the cell allows, in the extreme case, us to move from a discrete to a continuum model of the medium. The obtained results make it possible to establish the limits of the use of discrete representations of changes in the structure of materials and the continuum model of the propagation of acoustic vibrations in a medium.

\section{References}

[1] Aleksenko V.L.: Detection of acoustic-emission effects upon repeated loading of samples from steel St3sp. Technical diagnostics and non-destructive testing. International Scientific, Technical and Production Journal 4, 2017, 25-31.

[2] Capinteri A.: Structural damage diagnosis and lifetime assessment by acoustic emission monitoring. Engineering Fracture Mechanics 74, 2007, 273-289.

[3] Colombo S., Main I.G., Forde M.C.: Assessing damage of reinforced concrete beam using "b-value" analysis of acoustic emission signals. J. Mat. Civil Eng. ASCE. 15, 2003, 280-286.

[4] Golaski I., Gebski P., Ono K.: Diagnostics of rein for concrete bridges by acoustic emission. Journal of Acoustic Emission 1, 2002, 83-98.

[5] Jiang XU.: Space-time evolution rules study on acoustic emission location in rock under cyclic loading. Frontiers of Architecture and Civil Engineering in China 3(4), 2009, 422-427.

[6] Lu C., Mai Y-W., Shen Y-G.: Optimum information in cracking noise. Phys. Rev. E. 72, 2005, 027101-1.

[7] Marasanov V., Sharko A.: Discrete models characteristics of the forerunners of origin of the acoustic emission signals. IEEE First Ukraine Conference on Electrical and Computer Engineering, 2017

[8] Marasanov V., Sharko A.: Information-structural modeling of the the Forerunners of Origin of Acoustic Emission Signals in Nanoscale Objects. IEEE 38th International Conference on Electronics and Nanotechnology (ELNANO), 2018.

[9] Marasanov V.: Mathematical Models for Interrelation of Characteristics of the Developing Defects with Parameters of Acoustic Emission Signals. International Fronter Science Letters 10, 2016, 34-40.

[10] Marasanov V.V.: Energy spectrum of acoustic emission signals in complex media. Journal of Nano and Electronic Physics 4(9), 2017, 04024-1 - 04024-5.

[11] Paparo G., Gregori G.P., Coppa U., De Ritis R., Taloni A.: Acoustic Emission (AE) as a diagnostic tool in geophysics. Annals of Geophysics 2(45), 2002, 401-416.

[12] Petersen T.V., Botvina L.R.: Modeling of seismic activity by acoustic emission testing of metals. Geophys. Res. Abstr. 6, 2004, 07454.

[13] Pollok A.: Acoustic Emission Inspection. Metals Handbook. ASM International, 1989.

[14] Rundle J.B., Turcotte D.L., Shcherbakov R., Klein W., Sammis C.: Statistica physics approach to understanding the multiscale dynamics of earthquake fault systems. Rev. Geophys 41, 2003, 1-30.

[15] Shen Gongtian, Wu Zhanwen. Gongtian Shen: Study on Spectrum of Acoustic Emission Signals of Bridge Crane. Insight: Non-Destruct. Test. and Cond. Monit. 3(52), 2010, 144-147.

[16] Sundaresun M.I.: Linear location of acoustic emission sources with a single channel distributed sensor. Journal of intelledent material systems and structures 12, 2001, 689-699.

[17] Suzuki H., Kinto T., Taketo M., Ono K.: Appendix by Journal of Acoustik Emission 2 (vol. 14), 1996, 69-84.

\section{M.Sc. Artem Sharko}

e-mail: sharko_artem@ukr.net

Ph.D. graduate at Kherson National Technical University Faculty of Cybernetics and System of Engineering. Research: nondestructive testing, physical information technologies, acoustic emission. He has 16 research publications.

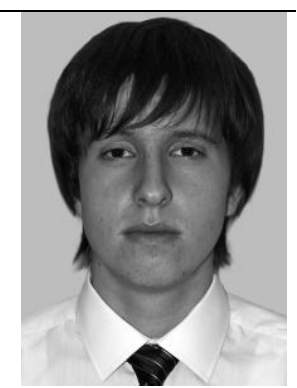

otrzymano/received: 25.08 .2018 